\title{
深圳市交通排放污染物浓度特征与影响因素
}

\author{
张晓春 ${ }^{1} ，$ 丘建栋 ${ }^{1}$ ，屈新明 ${ }^{1}$ ，徐炜铃 ${ }^{2} ， 丁 \quad$ 升 $^{3}$
}

1）深圳市城市交通规划设计研究中心有限公司，广东深圳 $518021 ； 2$ ) 东南大学交通学院, 江苏南京 211189;

3 ) 中山大学智能工程学院, 广东广州 510006

摘 要: 随着机动车保有量不断增加, 尾气污染问题日趋严重. 为掌握中国深圳市交通排放所带来的 路边空气污染状况, 探索交通与空气污染之间的关系, 设计了典型道路交通排放的路边监测实验, 以研究 空气污染中的交通排放规律和关键影响因素. 实验监测了典型道路的交通流、气象数据和空气污染物质量 浓度，通过对比监测点与空气质量监测国家控制点的数据，分析道路移动源对空气质量的影响：当交通流 量较小时, 路边监测点与空气质量监测国家控制点数据一致; 当交通流量 (特别是货运量) 大时, 路边监测 点的数据显著高于空气质量监测国家控制点的; 通过对 $\mathrm{NO}_{x}$ 和 $\mathrm{CO}$ 的质量浓度与主要影响因素的关联分析, 揭示了交通流量和气象对交通排放污染物质量浓度的影响, 排放污染物质量浓度整体趋势与总交通量较为 一致. 结果表明, 深圳市各区 $\mathrm{NO}_{x}$ 和 $\mathrm{CO}$ 质量浓度主要受到机动车 (尤其是货车) 排放的影响, 光照强度、 风向和风速对污染物的排放扩散有明显作用.

关键词: 环境工程; 尾气污染; 污染物质量浓度; 路边监测实验; 关联分析; 道路移动源; 交通排放 扩散; 交通流量; 光照强度; 氮氧化物; 一氧化碳

中图分类号：X511 文献标志码：A doi：10.3724/SP. J. 1249.2020.02178

\section{Characteristics and influencing factors of traffic pollutant emission concentration in Shenzhen City}

\author{
ZHANG Xiaochun ${ }^{1}$, QIU Jiandong ${ }^{1}$, QU Xinming ${ }^{1}$, \\ XU Weiling ${ }^{2}$, and DING Hui ${ }^{3}$
}

1) Shenzhen Urban Transport Planning Center Co. , Ltd. , Shenzhen 518021, Guangdong Province, P. R. China

2) School of Transport, Southeast University, Nanjing 211189, Jiangsu Province, P. R. China

3 ) School of Intelligent Systems Engineering, Sun Yat-Sen University, Guangzhou 510006, Guangdong Province, P. R. China

\begin{abstract}
With the increasing number of motor vehicles, exhaust pollution has become increasingly serious. In order to grasp the roadside air pollution caused by traffic emissions in Shenzhen City and explore the relationship between traffic and air pollution, we design a series of roadside monitoring experiments to study the law and key influencing factors of traffic emissions in air pollution. The experiments monitor the traffic flow, meteorological data, and air pollutant mass concentration of typical roads. By comparing the monitoring results with those of the national air quality control points, which reflects the impact of road moving sources on air quality, we find that when there is less traffic, the roadside monitoring results are consistent with those of the state control point; when the traffic flow
\end{abstract}

Received: 2019-09-17; Accepted: 2019-11-21

Foundation: National Key R\&D Program of China (2018YFB1601105); Special Funds for the Development of Strategic Emerging Industries of Shenzhen in the First Support Planning of 2017 (Shenzhen Development and Reform Commission (2017) No. 550)

Corresponding author: Professorate senior engineer ZHANG Xiaochun. E-mail: zhangxiaochun@ sutpc.com

Citation: ZHANG Xiaochun, QIU Jiandong, QU Xinming, et al. Characteristics and influencing factors of traffic pollutant emission concentration in Shenzhen City $[\mathbf{J}]$. Journal of Shenzhen University Science and Engineering, 2020, 37(2) : 178-186. (in Chinese) 
(especially freight) is large, the roadside monitoring results are significantly higher than those of the state control station. Based on the correlation analysis of nitrogen oxide $\left(\mathrm{NO}_{x}\right)$ mass concentration and carbon monoxide ( $\mathrm{CO}$ ) mass concentration with the main influencing factors, the effects of traffic flow and weather on the mass concentration and trend of pollutants emitted by traffic are revealed. The overall trend is more consistent with the total traffic volume. The results show that the mass concentration of $\mathrm{NO}_{x}$ and $\mathrm{CO}$ in various districts of Shenzhen are mainly affected by the emissions of motor vehicles ( especially trucks), and the light intensity, wind direction, and wind speed have a significant effect on the emission diffusion of pollutants.

Key words : environmental engineering; exhaust pollution; pollutant mass concentration; roadside monitoring experiment; correlation analysis; road moving source; traffic emission diffusion; traffic flow; light intensity; nitrogen oxide $\left(\mathrm{NO}_{x}\right)$; carbon monoxide

随着城市交通的快速发展和来自生产生活运输 需求的提升, 机动车保有量不断增加, 带来的交通 拥堵和尾气污染问题也日趋突出. 中国深圳市民用 汽车拥有量从 2013 年的 258.08 万辆增长到 2018 年 的 336.66 万辆 ${ }^{[1-2]}$, 机动车尾气排放成为城市空气 污染的主要来源之一. 研究表明, 机动车尾气排放 对北京、上海、广州和深圳等大中型城市空气污染 贡献在 $25 \%$ 以上 ${ }^{[3]}$, 严重影响城市空气质量, 且直 接危害人群健康 ${ }^{[4]}$. 因此, 针对城市交通尾气排放 以及交通污染问题的研究至关重要. 邱洪斌等 ${ }^{[5]}$ 于 2001 年对哈尔滨市一条主干道的大气颗粒物进行分 季度的监测和采样, 发现城市街道的大气颗粒物污 染严重, 气象条件是重要的影响因素. 谢敏等 ${ }^{[6]}$ 于 2008 年对广州市主干道附近的空气质量进行监测, 研究其大气污染物质量浓度的特征及影响因素, 结 果表明空气污染水平和污染物时空分布不均匀，以 及污染物质量浓度与气象因素之间有较高的多元线 性相关性.

为掌握深圳市交通排放所带来的路边空气污染 状况，探索交通与空气污染之间的关系，本研究设 计了深圳市交通碳排放实地监测实验. 选取南山 区、宝安区、福田区及罗湖区的典型道路交通场 景, 对路段交通流、气象数据和空气污染物质量浓 度进行了连续的监测调研, 分析了空气污染中的交 通排放贡献规律和关键影响因素.

\section{1 数据来源}

本次实地监测路段为深圳市南山区月亮湾大 道、宝安区新湖路段、福田区福华路和罗湖区文锦 中路. 其中, 月亮湾大道对照空气质量监测国家控 制点南海子站, 所选监测点位为双向 8 车道, 监测

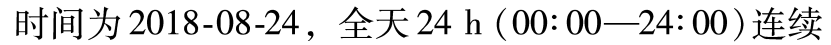
监测; 宝安区新湖路段对照空气质量监测国家控制 点西乡站, 所选监测点位为双向 3 车道, 监测时间 段为 2018-08-22-2018-08-23, 共计 $24 \mathrm{~h}$ 连续监 测; 福田区福华路对照空气质量监测国家控制点通 心岭子站, 所选监测点位为双向 8 车道, 监测时间 为 2018-08-24, 全天 $24 \mathrm{~h}(00: 00-24: 00)$ 连续监 测; 罗湖区文锦中路对照空气质量监测国家控制点 洪湖站, 所选监测点位为双向 10 车道, 实验时间 段为 2018-08-25-2018-08-26, 共计 $24 \mathrm{~h}$ 连续监 测. 实验监测点和空气质量监测国家控制点分布如 图 1. 实验监测现场图和实验监测点信息请扫描论 文末页右下角二维码查看图 S1 和表 S1.

本实验利用车载污染自动监测系统、化学发光 法 $\mathrm{NO}-\mathrm{NO}_{2}-\mathrm{NO}_{x}$ 分析仪、红外相关法 $\mathrm{CO}$ 分析仪、 紫外光度法 $\mathrm{O}_{3}$ 分析仪和 $\beta$ 射线法颗粒物 $\left(\mathrm{PM}_{2.5}\right.$ 或 $\mathrm{PM}_{10}$ ) 监测仪, 分别对 $\mathrm{NO}_{x} 、 \mathrm{CO} 、 \mathrm{O}_{3}$ 、可吸入颗粒 物 $\mathrm{PM}_{10}$ 和细颗粒物 $\mathrm{PM}_{2.5}$ 污染物的质量浓度进行 24 $\mathrm{h}$ 的连续监测, 并同步采集道路交通车流、气象数 据以及空气质量监测国家控制点数据信息.

\section{2 数据监测概况}

\section{1 典型路段交通流监测}

交通流量是表征车辆活动水平的重要参数, 也 是影响机动车排放量的重要参数. 考虑到监测设备 和道路情况等因素, 本研究采用视频录像法, 结合 人工计数, 采集实验监测道路的交通数据, 获取分 车辆类型（按车辆排放因子类型划分）的小时流量 数据. 夜间采用抽样调查法, 每小时采集车流数据 时长为 $20 \mathrm{~min}$. 


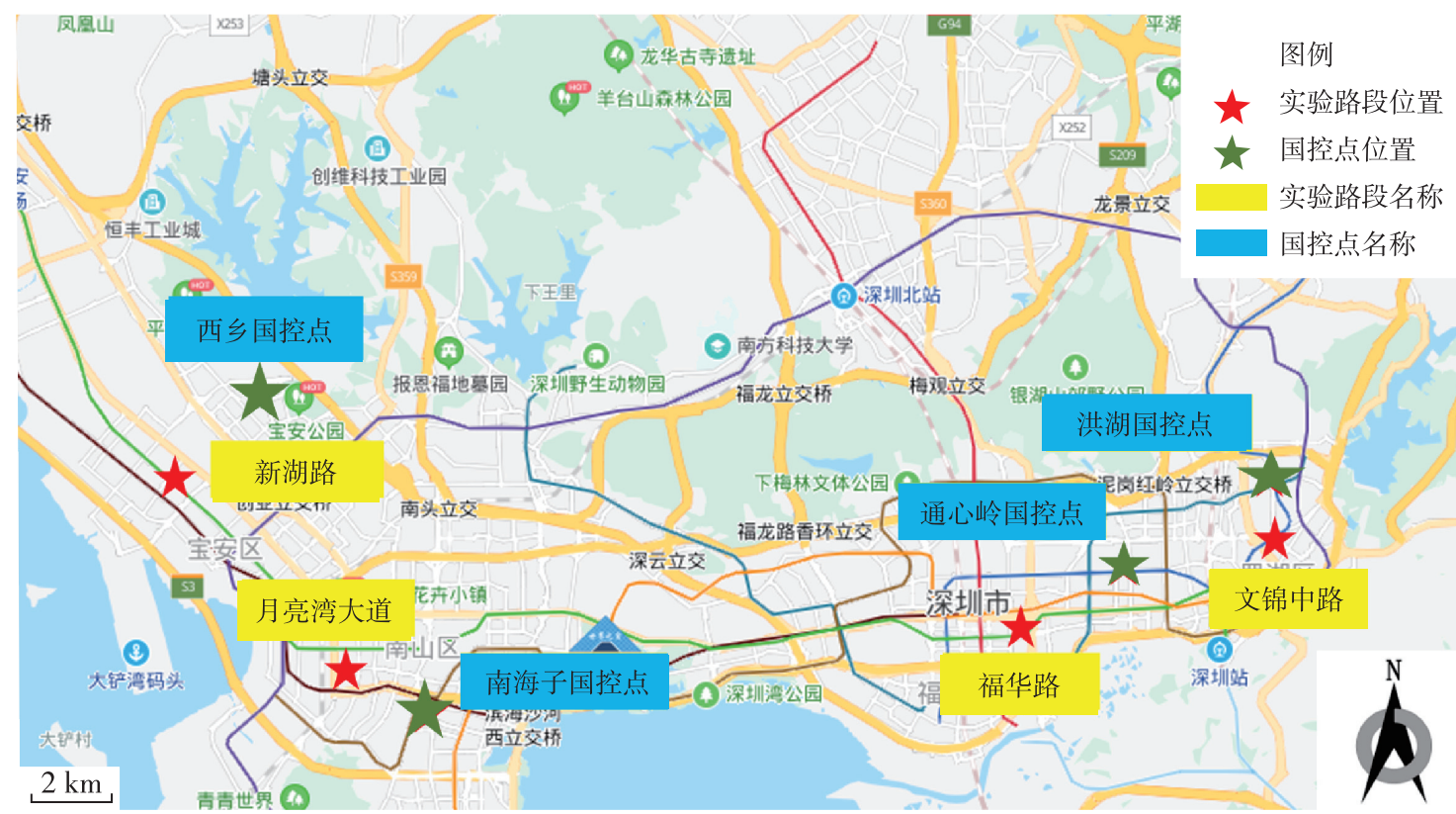

图 1 实验监测点和空气质量监测国家控制点分布

Fig. 1 Overall distribution map of experimental monitoring points and air quality control points

\section{2 空气污染物监测}

将实验监测车行驶至预设的路边监测地点，分 别在南山区月亮湾大道、宝安区新湖路、福田区福 华路和罗湖区文锦中路采取定点测量的方式进行监 测. 每个监测点位连续测量 $24 \mathrm{~h}$.

\section{3 气象数据监测}

气象数据的监测采用车载式空气质量自动监测 系统，设定采样周期为 $1 \mathrm{~min} ，$ 测量数据包括温度、 海平面气压、平均风速、平均风向和相对湿度等指
标. 这些气象数据将作为交通环境污染物质量浓度 影响因素进行关联性分析.

\section{3 实验结果与分析}

\section{1 监测结果与空气质量监测国家控制点对比}

将路边监测实验的实测结果与对应空气质量监 测国家控制点数据进行对比分析. 质量浓度的日均 值及小时变化的对比结果分别如表 1 和图 2 .

表 1 深圳市实验监测点与空气质量监测国家控制点主要污染物质量浓度的日均值

Table 1 Daily average value of major pollutants concentration in Shenzhen experimental monitoring points and air quality control points

\begin{tabular}{|c|c|c|c|c|c|c|c|}
\hline 监测点 & $\begin{array}{l}\text { 首要 } \\
\text { 污染物 }\end{array}$ & $\begin{array}{c}\text { 空气质量 } \\
\text { 指数 }\end{array}$ & $\begin{array}{l}\rho\left(\mathrm{PM}_{2.5}\right) / \\
\left(\mathrm{mg} \cdot \mathrm{m}^{-3}\right)\end{array}$ & $\begin{array}{l}\rho\left(\mathrm{PM}_{10}\right) / \\
\left(\mathrm{mg} \cdot \mathrm{m}^{-3}\right)\end{array}$ & $\begin{array}{l}\rho\left(\mathrm{NO}_{2}\right) / \\
\left(\mathrm{mg} \cdot \mathrm{m}^{-3}\right)\end{array}$ & $\begin{array}{l}\rho(\mathrm{CO}) / \\
\left(\mathrm{mg} \cdot \mathrm{m}^{-3}\right)\end{array}$ & $\begin{array}{c}\rho\left(\mathrm{O}_{3} \text { 日 } \max 8 \mathrm{~h}\right)^{1)} / \\
\left(\mathrm{mg} \cdot \mathrm{m}^{-3}\right)\end{array}$ \\
\hline 南山月亮湾大道 & $\mathrm{PM}_{2.5}$ & 129 & 98.1 & 172.4 & 84.6 & 1.9 & 75.0 \\
\hline 南海子站 & $\mathrm{O}_{3}$ & 151 & 37.0 & 57.0 & 42.5 & 0.9 & 215.6 \\
\hline 宝安新湖路 & $\mathrm{O}_{3}$ & 126 & 45.4 & 62.9 & 29.1 & 1.1 & 188.4 \\
\hline 西乡站 & $\mathrm{O}_{3}$ & 91 & 27.5 & 55.8 & 41.8 & 0.6 & 148.4 \\
\hline 福田福华路 & $\mathrm{O}_{3}$ & 167 & 57.7 & 84.1 & 36.4 & 1.7 & 231.6 \\
\hline 通心岭子站 & $\mathrm{O}_{3}$ & 203 & 51.5 & 65.2 & 27.5 & 0.9 & 276.8 \\
\hline 罗湖文锦中路 & $\mathrm{PM}_{10}$ & 113 & 49.4 & 174.0 & 25.6 & 1.5 & 149.8 \\
\hline 洪湖站 & $\mathrm{O}_{3}$ & 95 & 44.1 & 65.3 & 35.9 & 0.9 & 153.0 \\
\hline
\end{tabular}

1) $\rho\left(\mathrm{O}_{3}\right.$ 日 $\left.\max 8 \mathrm{~h}\right)$ 为 $\mathrm{O}_{3}$ 日最大 $8 \mathrm{~h}$ 平均质量浓度, 即连续 $8 \mathrm{~h} \mathrm{O}_{3}$ 质量浓度最大平均值 


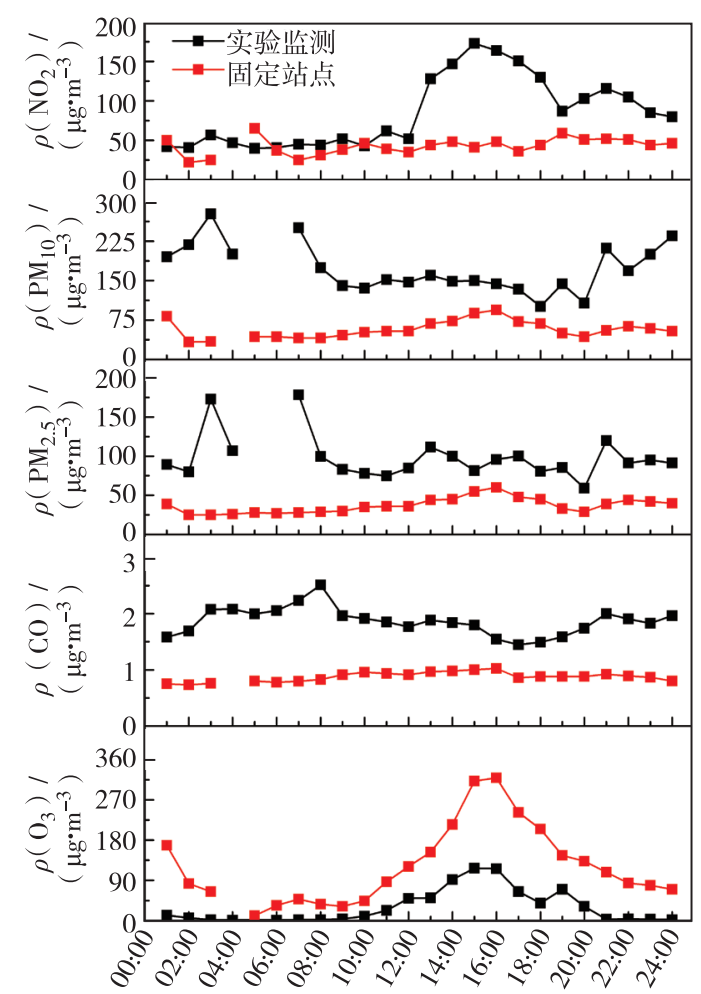

时刻

( a ) 南山区

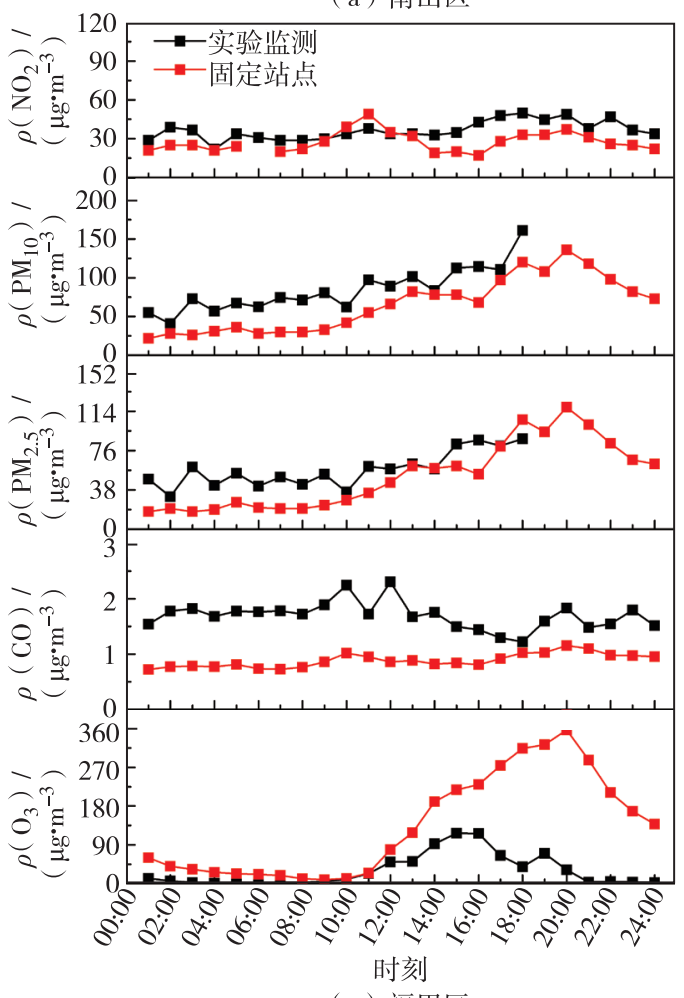

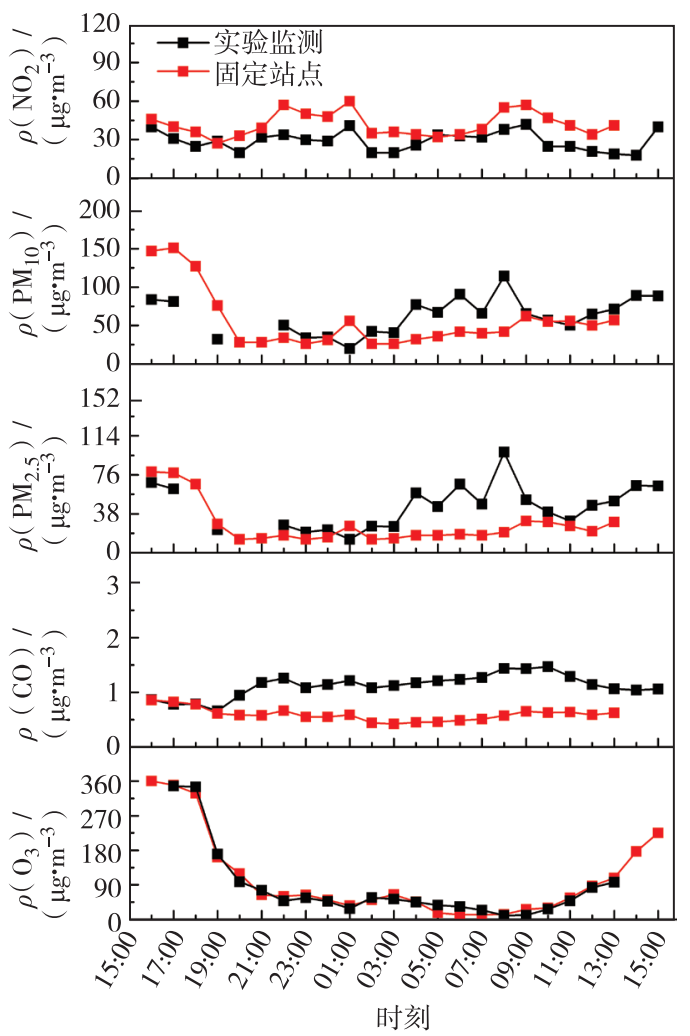

(b) 宝安区

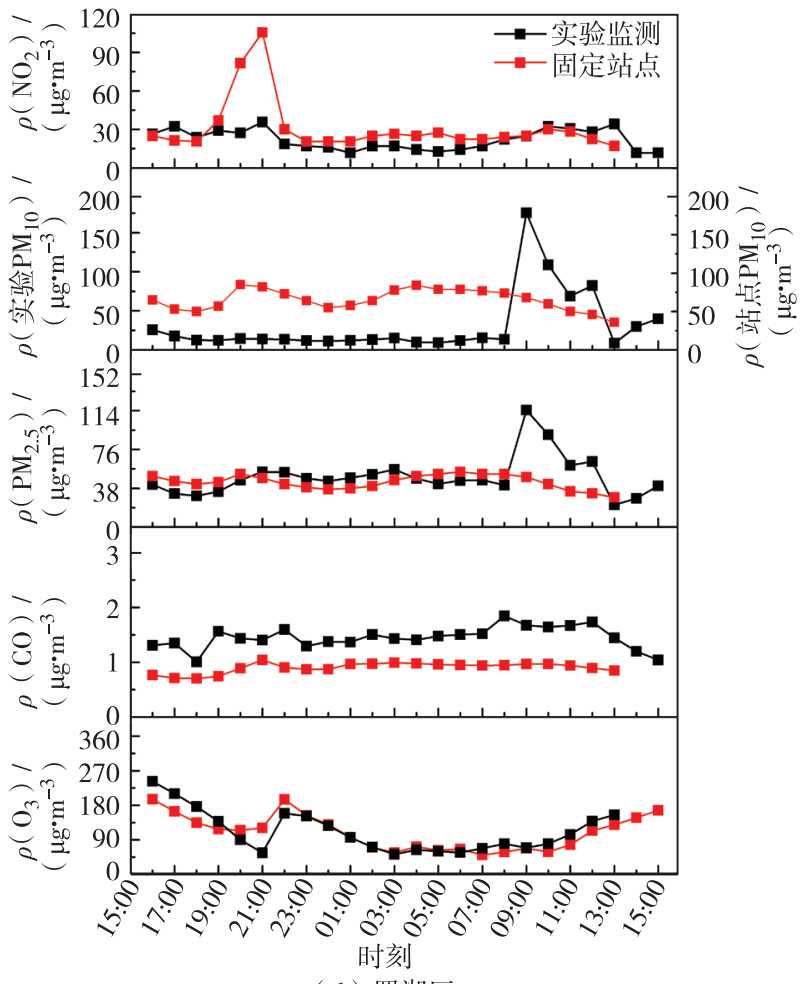

(d) 罗湖区

图 2 深圳市实验监测点与空气质量监测国家控制点主要污染物小时变化

Fig. 2 Hourly variation of main pollutants in Shenzhen experimental monitoring points and air quality control points

由表 1 和图 2 可见，深圳市路边监测到的首要 污染物与附近空气质量监测国家控制点的较一致,
监测期间主要为 $\mathrm{O}_{3}$ 污染. 整体上, 深圳市路边监 测到的 $\rho\left(\mathrm{O}_{3}\right)$ 趋势与附近监测站的最为一致, 均在 
下午达到峰值，其余时间处于较低水平，而其他污 染物在不同主干道差异有所区别. 其中，污染物在 南山区月亮湾大道路边监测小时质量浓度趋势及大 小均与监测站的差异最大; $\mathrm{NO}_{2}$ 和颗粒物的质量浓 度在宝安区新湖路、福田区福华路及罗湖区文锦中 路变化趋势及大小均较为一致, 而路边监测的 $\rho(\mathrm{CO})$ 则波动相对更大且值较大, 空气质量监测国 家控制点 $\mathrm{CO}$ 的小时质量浓度变化幅度则较小，基 本比较平稳.

深圳市南山区月亮湾大道实验监测的小时变化 结果中, 各种空气污染物在路边监测小时质量浓度 大小均与监测站的差异大: 除 $\mathrm{O}_{3}$ 以外, 其余空气 污染物日均值质量浓度均显著高于空气质量监测国 家控制点南海子站, 实验监测 $\rho\left(\mathrm{PM}_{2.5}\right)$ 为南海子 站的 2.7 倍; $\rho\left(\mathrm{PM}_{10}\right)$ 为站点的 3.0 倍; $\rho\left(\mathrm{NO}_{2}\right)$ 为 南海子站的 2.0 倍; $\rho(\mathrm{CO})$ 为南海子站 2.1 倍; 而 南海子站监测的 $\rho\left(\mathrm{O}_{3}\right)$ 则为实验的 2.9 倍. 且除 $\mathrm{O}_{3}$ 以外, 其余污染物质量浓度变化趋势与空气质量监 测国家控制点南海子站也不一致：月亮湾大道路边 监测得到的首要污染物为 $\mathrm{PM}_{2.5}$; 南海子站则为 $\mathrm{O}_{3}$; 空气质量指数均处于轻度污染水平. 实验监测的 $\rho\left(\mathrm{O}_{3}\right)$ 小时变化趋势与国家控制站点较为一致，但 其他污染物出现多个峰值情况, 与站点监测情况差 别较大. 南山区月亮湾大道作为深圳市主要货运通 道, 承担了大量货车输运, 因此, 交通排放污染更 为严重, 这是南山区路边监测实验结果多项空气污 染物高于监测站的主要原因. 路边监测的 $\rho\left(\mathrm{O}_{3}\right)$ 显 著低于站点, 与道路移动源所排放的前体物 $\mathrm{NO}_{x}$ 和 挥发性有机化合物（volatile organic compounds, $V O C s)$ 的比例直接相关. 由于 $\mathrm{O}_{3}$ 大部分是二次污 染物, 需要前驱物 $\mathrm{NO}_{x}$ 和挥发性有机物在阳光照射 下，通过光化学反应产生; 在货车交通量大的月亮 湾大道, 机动车排放的空气污染物中, 一些成分可 以与 $\mathrm{O}_{3}$ 产生化学反应, 减少 $\mathrm{O}_{3}$ 的产生; 而 $\mathrm{O}_{3}$ 其 他的前驱物随风输送到郊区，重新形成 $\mathrm{O}_{3}$, 导致郊 区 $\rho\left(\mathrm{O}_{3}\right)$ 高于市区 ${ }^{[7]}$.

在深圳市宝安区新湖路实验监测的小时变化结 果中, $\rho\left(\mathrm{NO}_{2}\right)$ 与 $\rho\left(\mathrm{O}_{3}\right)$ 的数值大小、变化趋势与空 气质量监测国家控制点西乡站高度一致; 除 03:00- 07:00 期间出现不一致的峰值波动, $\rho\left(\mathrm{PM}_{2.5}\right) 、 \rho\left(\mathrm{PM}_{10}\right)$ 的数值大小整体趋势与西乡站 较一致; 实验监测的 $\rho(\mathrm{CO})$ 有不明显的峰值, 但 西乡站的 $\rho(\mathrm{CO})$ 小时变化不明显，且质量浓度大 小显著低于实验监测值. 实验监测点与西乡站具体
对比如下：除 $\mathrm{NO}_{2}$ 以外, 实验监测的空气污染物质 量浓度均高于西乡站的; 实验监测的 $\rho\left(\mathrm{PM}_{2.5}\right)$ 比 站点的高 $65.1 \% ; \rho\left(\mathrm{PM}_{10}\right)$ 比站点的高 $12.7 \%$; $\rho(\mathrm{CO})$ 为站点的 $83.3 \% ; \rho\left(\mathrm{O}_{3}\right)$ 比站点的高 $27.0 \%$; 而站点监测的 $\rho\left(\mathrm{NO}_{2}\right)$ 比实验监测的高 $43.6 \%$. 新湖路路边监测得的首要污染物与西乡站 一致，均为 $\mathrm{O}_{3}$; 新湖路路边监测空气质量指数处于 轻度污染水平, 西乡站空气质量指数则处于良好水 平. 实验的新湖路段作为宝安区次干路，路段交通 量较小，道路带来的交通排放污染相对少，这是新 湖路路边监测实验结果多项空气污染物与监测站较 为一致的主要原因.

深圳市福田区福华路实验监测的小时变化结果 中, $\rho\left(\mathrm{NO}_{2}\right) 、 \rho\left(\mathrm{PM}_{2.5}\right)$ 与 $\rho\left(\mathrm{PM}_{10}\right)$ 的数值大小、变 化趋势与空气质量监测国家控制点通心岭子站高度 一致; 实验监测的 $\rho\left(\mathrm{O}_{3}\right)$ 与空气质量监测国家控制 点的变化趋势较一致, 但空气质量监测国家控制点 峰值明显高于实验监测的，且峰值水平持续时间更 长; 实验监测的 $\rho(\mathrm{CO})$ 有不明显的峰值, 但空气 质量监测国家控制点小时变化不明显，且质量浓度 大小显著低于实验监测值. 实验监测点与空气质量 监测国家控制点具体对比如下: 实验监测 $\rho\left(\mathrm{PM}_{2.5}\right)$ 高于站点 $12.0 \% ; \rho\left(\mathrm{PM}_{10}\right)$ 高于站点 $29.0 \%$; $\rho\left(\mathrm{NO}_{2}\right)$ 高于站点 $32.4 \% ; \rho(\mathrm{CO})$ 高于站点 $88.9 \%$; 而站点 $\rho\left(\mathrm{O}_{3}\right)$ 高于实验监测 $19.5 \%$. 福华路路边监 测得到的首要污染物与通心岭子站一致，均为 $\mathrm{O}_{3}$; 福华路边监测空气质量指数处于轻度污染水平, 通 心岭子站空气质量指数则处于中度污染水平.

深圳市罗湖区文锦中路实验监测的小时变化结 果中, $\rho\left(\mathrm{O}_{3}\right)$ 在变化趋势及大小上与洪湖站空气质 量监测国家控制点高度一致; 实验监测的 $\rho\left(\mathrm{NO}_{2}\right)$ 与空气质量监测国家控制点的趋势与大小较为一 致, 但受到附近出现短时污染的影响, 空气质量监 测国家控制点在 19:00-20:00 出现异常高的峰值; 实验监测的 $\rho(\mathrm{CO})$ 有不明显的峰值, 但空气质量 监测国家控制点小时变化不明显，且质量浓度大小 显著低于实验监测. 对于实验监测的颗粒物情况, 除了受到监测点附近房屋拆迁造成的短时扬尘污染 影响外, 其余时间 $\rho\left(\mathrm{PM}_{2.5}\right)$ 的数值大小、变化趋 势与空气质量监测国家控制点的较一致; $\rho\left(\mathrm{PM}_{10}\right)$ 实验监测大小显著低于空气质量监测国家控制点. 实验监测点与空气质量监测国家控制点具体对比如 下: 实验监测 $\rho\left(\mathrm{PM}_{2.5}\right)$ 高于站点 $12.0 \% ; \rho\left(\mathrm{PM}_{10}\right)$ 为站点 2.7 倍; $\rho(\mathrm{CO})$ 高于站点 $66.7 \%$; 而站点 
$\rho\left(\mathrm{NO}_{2}\right)$ 高于实验监测的 $40.2 \%$; 站点 $\rho\left(\mathrm{O}_{3}\right)$ 高于 实验监测的 $2.1 \%$. 罗湖区文锦中路路边监测得到 的首要污染物为 $\mathrm{PM}_{10}$, 洪湖站则为 $\mathrm{O}_{3}$, 路边监测 空气质量指数处于轻度污染水平, 洪湖站则处于良 好水平.

\section{2 检测结果与主要影响因素关联分析}

3.2.1 $\rho\left(\mathrm{NO}_{x}\right) 、 \rho\left(\mathrm{O}_{3}\right)$ 与主要影响因素关联分析 由以上分析可知，各主干道路边监测的 $\rho\left(\mathrm{NO}_{x}\right)$ 小时变化显著受到交通排放的影响，均呈 现早晚高峰的特征，更好地反映了道路移动源对空

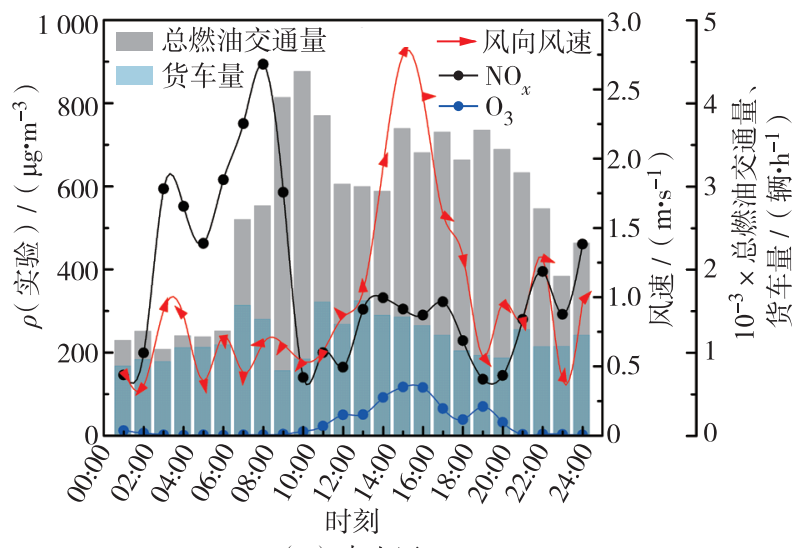

(a) 南山区

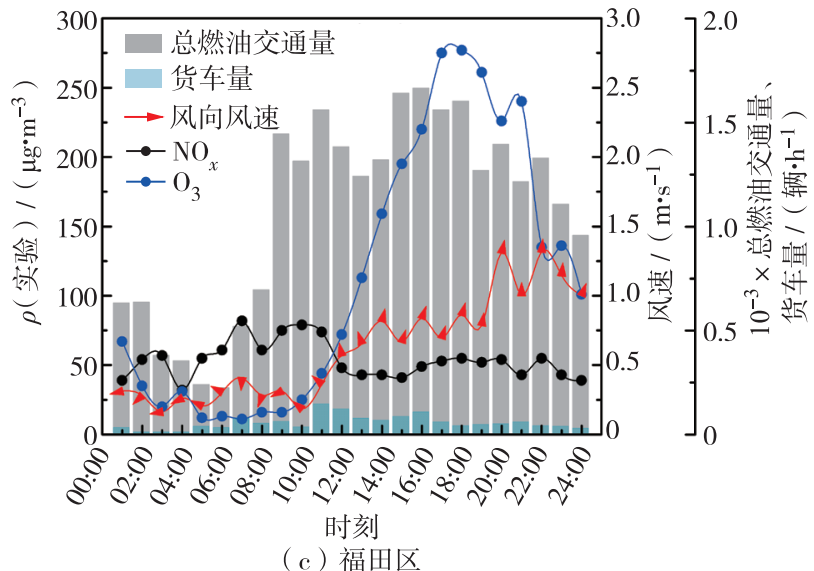

气质量的影响. 此外, 2015 年深圳市机动车 $\mathrm{NO}_{x}$ 排放量为 $7.9 \times 10^{4} \mathrm{t}$, 占全市 $\mathrm{NO}_{x}$ 总排放量的 $69 \%$, 可见 $\mathrm{NO}_{x}$ 主要来源于机动车的尾气排放. 同 时 $\mathrm{NO}_{x}$ 作为 $\mathrm{O}_{3}$ 主要前驱物之一, $\rho\left(\mathrm{NO}_{x}\right)$ 受到相关 光化学反应等的显著影响 ${ }^{[8-9]}$. 实验期间, 风向风 速是最为多变的气象因素，对污染物的扩散有明显 作用. 为进一步认识交通与路边污染的影响关系, 将路边监测实验的 $\rho\left(\mathrm{NO}_{x}\right)$ 与 $\rho\left(\mathrm{O}_{3}\right)$ 的实测结果、 路边实测交通总量以及风向风速情况进行对比分 析, 如图 3.

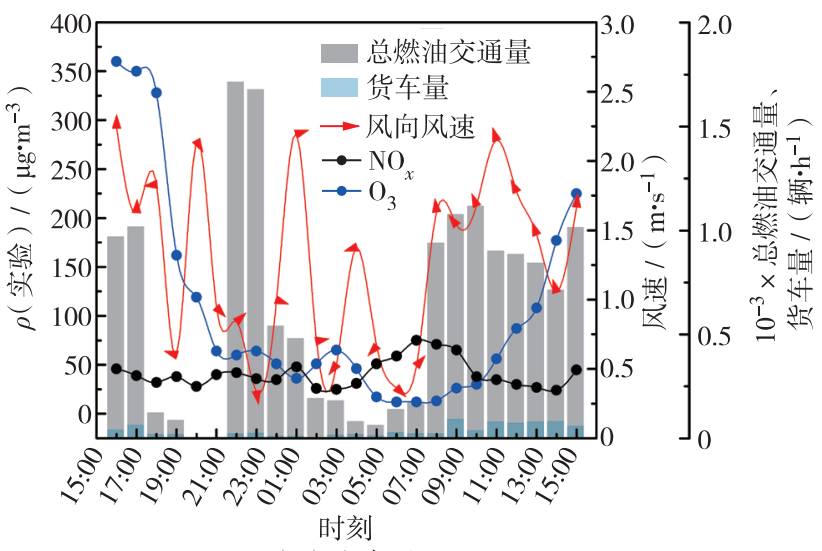

(b) 宝安区

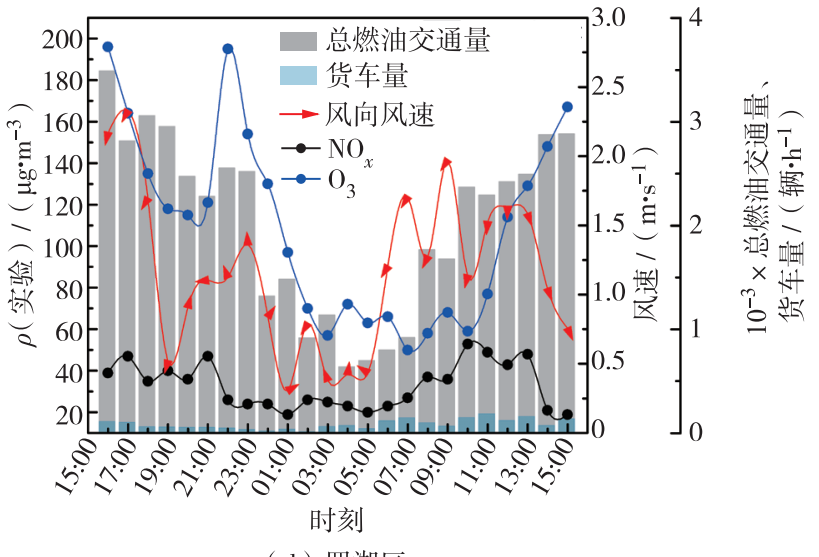

(d) 罗湖区

图 3 深圳市实验监测点 $\rho\left(N_{x}\right) 、 \rho\left(O_{3}\right)$ 与主要影响因素关联分析

Fig. 3 Correlation analysis of $\rho\left(\mathrm{NO}_{x}\right) 、 \rho\left(\mathrm{O}_{3}\right)$ and main influencing factors at experimental monitoring points of Shenzhen

南山区月亮湾大道的 $\mathrm{NO}_{x}$ 主要在 02:00-07:00 处于最高峰值水平, $12: 00-16: 00$ 处于次高峰水 平, 并且在 21:00 后 $\rho\left(\mathrm{NO}_{x}\right)$ 重新上升, 主要受到 交通排放和气象因素的共同影响. 凌晨 $00: 00-$ 05:00内，南山区交通量明显下降，但货车量仍维 持在较高水平, 与白天较接近, 并且货车占机动车 排放的 $\mathrm{NO}_{x}$ 的分担率高, 因此保持的较大货车量仍 带来较大的 $\mathrm{NO}_{x}$ 排放量；监测期间风速较低，接近
静风状态，同时夜间相对湿度高，边界层高度低， 形成较差的扩散条件，使得 $\mathrm{NO}_{x}$ 难以扩散，进一步 积累，两个条件结合下导致了 02:00-07:00 时间段 $\rho\left(\mathrm{NO}_{x}\right)$ 最高峰值水平的情况. 12:00-16:00 时间 段月亮湾大道交通量处于较高水平并且货车量维持 在峰值状态, $\rho\left(\mathrm{NO}_{x}\right)$ 达到次高峰水平，但远低于凌 晨的最高峰水平, 这主要是由于下午日照辐射强度 加强, 促进了二次污染物 $\mathrm{O}_{3}$ 的形成, 消耗了 $\mathrm{NO}_{x}$, 
同时期间风速及边界层高度均较高，利于污染物的 水平及垂直方向上的扩散，进一步降低了 $\rho\left(\mathrm{NO}_{x}\right)$ 水平 ${ }^{[10]}$. 此外, 在交通早高峰期间 $(08: 00-$ 10:00)，监测到的氮氧化物反而明显下降，可能由 于货车作为其主要污染贡献源，在期间达到全日最 低水平, 轻型客车占主要的交通量, 同时日间开始 扩散条件转好，日照辐射强度增强，使得 $\rho\left(\mathrm{NO}_{x}\right)$ 迅速降低 ${ }^{[11]}$.

宝安区新湖路的 $\rho\left(\mathrm{NO}_{x}\right)$ 在 04:00-08:00 处于 明显峰值水平, 监测日内主要受到气象因素的影 响. 从 17:00 开始至凌晨有持续降雨过程, 对 $\mathrm{NO}_{x}$ 有清除作用，同时风速较高，有利于其水平扩散， 使得 $\rho\left(\mathrm{NO}_{x}\right)$ 相对稳定且较低, 受到交通量影响小. 在 04:00-08:00, 风速有所降低，不再有降雨过 程, 大气相对湿度较高, 扩散条件转差, $\mathrm{NO}_{x}$ 重新 开始累积, 并随着早高峰期间的较高交通排放量而 达到峰值状态.

福田区福华路的 $\rho\left(\mathrm{NO}_{x}\right)$ 在 06:00-10:00 达到 最高峰值水平, 且在 $01: 00-02: 00$ 及 $15: 00-21: 00$ 维持在较高值, 处于次峰值水平, 主要受到气象因 素及交通排放的共同影响. 在 06:00-10:00 风速尤 其低，接近静风状态，不利于扩散; 日照辐射强度 仍较弱, 不利于 $\mathrm{O}_{3}$ 形成，减少 $\mathrm{NO}_{x}$ 的消耗; 同时 交通量开始不断增加, 至早高峰的峰值水平, $\mathrm{NO}_{x}$ 排放不断增加和累积, 达到日最高峰值水平. 在 01:00-02:00, 主要受到气象因素的影响, 风速极 低，边界层高度低，整体的水平与垂直扩散条件不 佳，相对湿度高，且 $\mathrm{O}_{3}$ 在夜间不利于生成，使得 在较低的交通排放条件下, $\mathrm{NO}_{x}$ 维持在较高值 ${ }^{[12]}$. 在 $15: 00-21: 00, \rho\left(\mathrm{NO}_{x}\right)$ 较高主要受到交通排放的 影响，期间交通量一直处于高值状态，但由于风速 有所增加, 扩散条件转好, 未达到最高峰值水平.

罗湖区文锦中路的 $\mathrm{NO}_{x}$ 在 16:00-22:00 及次日 07:00-12:00 维持在较高水平的状态, 且监测到的 $\rho\left(\mathrm{NO}_{x}\right)$ 水平在日间高于夜间, 主要受到交通排放 变化的影响. 在 16:00-22:00 及次日 07:00-12:00 路边监测的交通量处于两个峰值水平, 交通排放 大, $\rho\left(\mathrm{NO}_{x}\right)$ 增加. 夜间交通量较少, 并且二次污染 物 $\mathrm{O}_{3}$ 异常地维持在较高水平，甚至出现了与下午 日照辐射强度大时类似的高值水平，是夜间 $\rho\left(\mathrm{NO}_{x}\right)$ 水平较低的主要原因 ${ }^{[13]}$.

\subsection{2 $\rho(\mathrm{CO})$ 与主要影响因素关联分析}

同样, 各主干道路边监测 $\rho(\mathrm{CO})$ 的小时变化 受到交通排放的影响，具有早晚高峰的特征，一定
程度上反映了道路移动源对空气质量的影响. 相关 研究 ${ }^{[14]}$ 表明, $\mathrm{CO}$ 主要来源于燃煤及机动车尾气. 为进一步认识交通与路边污染的影响关系，将路边 实测的 $\rho(\mathrm{CO})$ 结果、路边实测交通总量以及风向 风速情况进行对比分析，结果如图 4.

南山区月亮湾大道的 CO 在 02:00-07:00 处于 较高质量浓度水平，在 07:00 达到第 1 个峰值，在 20:00达到第 2 个峰值, 其质量浓度主要受交通排放 和气象因素的共同影响. 00:00-05:00 内，月亮湾 大道交通量明显下降，但货车量仍维持在较高水 平, 与白天较为接近, 并且货车占机动车排放的 $\mathrm{CO}$ 分担率高, 因此较大货车量的保持仍带来较多 的 CO 排放量; 监测期间风速较低，接近静风状态， 同时夜间相对湿度高，边界层高度低，扩散条件 差, 使得 $\mathrm{CO}$ 难以扩散，并进一步积累，两个条件 结合下导致了 $\rho(\mathrm{CO})$ 在 02:00-07:00 维持高水平. 20:00 $\rho(\mathrm{CO})$ 再次出现峰值，与较高交通量和夜间 逆温层增加导致的污染物积累相关. 12:00-16:00 月亮湾大道交通量处于较高水平，并且货车量维持 在峰值状态, $\rho(\mathrm{CO})$ 远低于凌晨的最高峰水平, 这 主要是由于下午日照辐射强度加强, 促进了二次污 染物 $\mathrm{O}_{3}$ 的形成，消耗了前提物 $\mathrm{CO}$; 同时期间风速 及边界层高度均较高, 利于污染物在水平及垂直方 向上的扩散，进一步降低了 $\rho(\mathrm{CO})$ 水平. 在交通 早高峰期间 $(08: 00-10: 00)$, 监测到的 $\rho(\mathrm{CO})$ 明 显下降，这是由于作为其主要污染贡献源的货车, 在此期间达到全日最低水平，轻型客车占主要的交 通量，同时从日间开始，扩散条件转好，日照辐射 强度增强

宝安区新湖路的 $\rho(C O)$ 在 07:00-09:00 出现 第 1 个峰值, 在 21:00 左右出现第 2 个峰值, 监测 日内主要受到交通排放变化的影响. 从17:00开始 至 00:00 有持续降雨过程, 但对 $\rho(\mathrm{CO})$ 影响较小, 随着降雨减少，道路交通排放再次增加, $\rho(\mathrm{CO})$ 在 21:00 左右达到峰值水平. 在 07:00-09:00为交通 高峰期, $\rho(\mathrm{CO})$ 受交通排放影响显著, 达到峰值 水平.

福田区福华路的 $\rho(\mathrm{CO})$ 在 08:00-11:00 达到 最高峰值水平, 在 19:00-22:00 处于次峰值水平, 主要受到交通排放的影响. 在 $08: 00-11: 00$ 交通量 为早高峰的峰值水平, 交通排放大, $\rho(\mathrm{CO})$ 达到峰 值水平; 同时风速尤其低，基本接近静风状态，不 利于扩散，CO 污染物不断增加和累积，达到日最 高小时质量浓度值. 在 19:00-22:00, $\rho(\mathrm{CO})$ 较高 


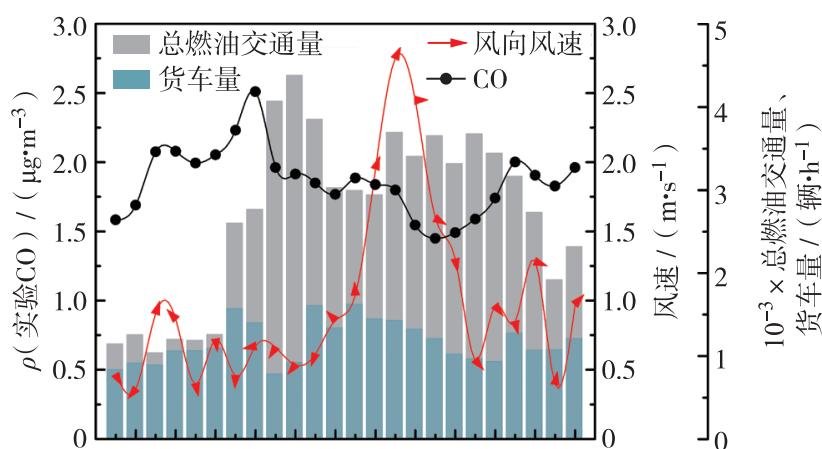

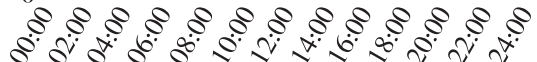

时刻

( a ) 南山区

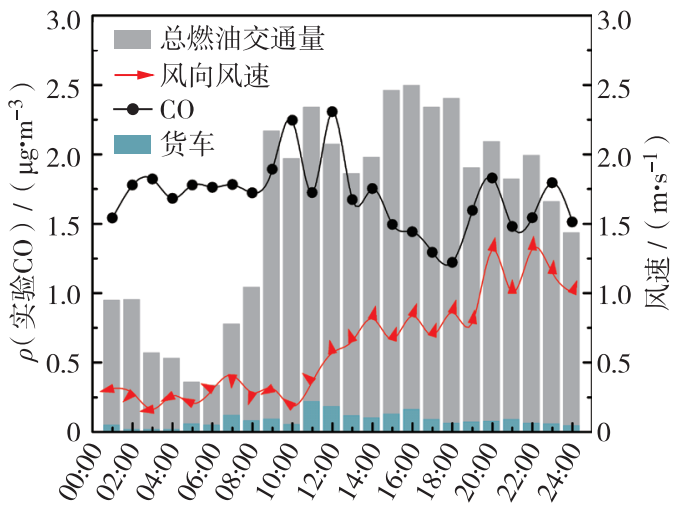

时刻

(c) 福田区

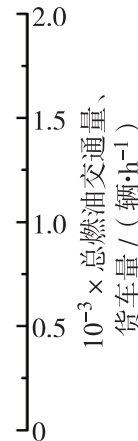

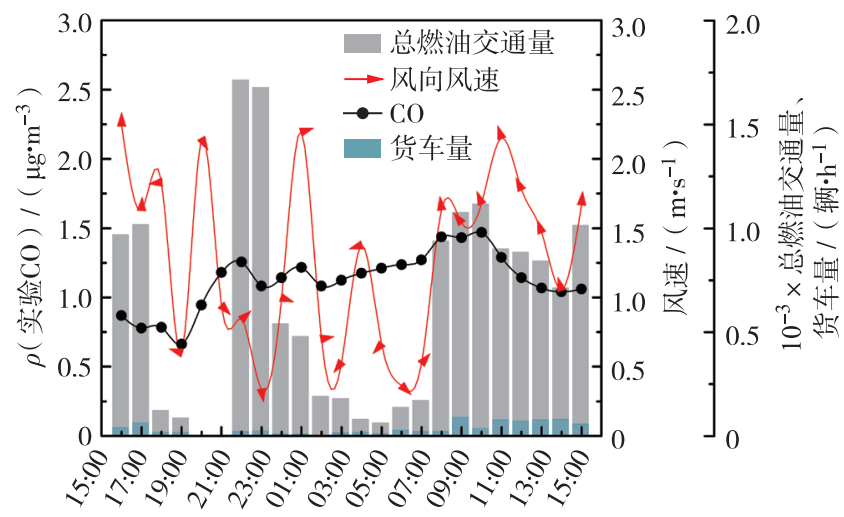

时刻

(b) 宝安区

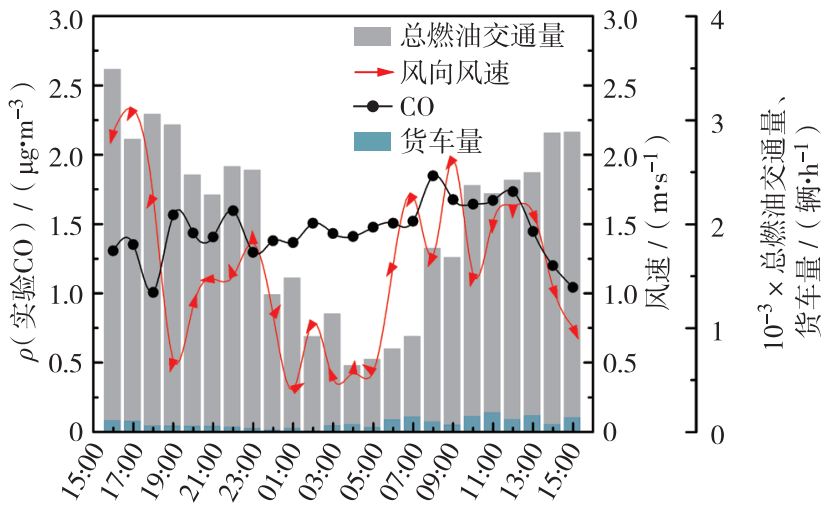

时刻

(d) 罗湖区

图 4 深圳市实验监测点 $\rho(\mathrm{CO})$ 与主要影响因素关联分析图

Fig. 4 Correlation analysis of $\rho$ ( $\mathrm{CO}$ ) and main influencing factors at experimental monitoring points of Shenzhen

主要是受到交通排放的影响，期间交通量一直处于 高值状态，虽然期间风速有所增加，扩散条件转 好, 但 $\rho(\mathrm{CO})$ 也明显增加至次峰值水平.

罗湖区文锦中路的 $\rho(\mathrm{CO})$ 在 07:00 出现最高峰 值, 并在 $08: 00-11: 00$ 维持在较高水平,在 18: 00-21:00 出现次峰值质量浓度, 主要受到交通 排放变化的影响. 在 07:00 和 18:00-21:00 路边监 测的交通量处于较高水平，交通排放大, $\rho(\mathrm{CO})$ 增 加. 00:00-06:00 虽然交通量较小，但风速低，边 界层高度低，逆温层增加，整体上水平与垂直扩散 条件不佳, $\mathrm{CO}$ 污染物不断积累, $\rho(\mathrm{CO})$ 维持在较

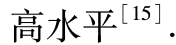

\section{4 结 论}

1）深圳市主干道路边监测的污染物小时质量 浓度大小和趋势能够反映道路移动源对空气质量的
影响. 当交通流量较少时，路边监测结果与空气质 量监测国家控制点保持一致; 当交通流量 (特别是 货运车量) 大时，路边监测结果显著高于空气质量 监测国家控制点.

2) 深圳市主干道路边监测的 $\rho\left(\mathrm{NO}_{x}\right)$ 和 $\rho(\mathrm{CO})$ 主要受到机动车排放和气象因素的影响, 整 体趋势与总交通量较为一致.

基金项目：国家重点研发计划资助项目（2018YFB1601105); 深圳 市战略性新兴产业发展专项资金资助项目 (深发改 〔2017〕550 号)

作者简介：张晓春 (1973-), 深圳市城市交通规划设计研究中心 有限公司教授级高级工程师. 研究方向: 交通规划、智 能交通和交通排放.E-mail:zhangxiaochun@ sutpc.com

引 文: 张晓春, 丘建栋, 屈新明, 等. 深圳市交通排放污染物 浓度特征与影响因素分析 $[\mathrm{J}]$. 深圳大学学报理工版, $2020,37(2)$ : 178-186. 


\section{参考文献 / References :}

[ 1 ] 深圳市统计局. 深圳市 2013 年国民经济和社会发展统 计公报 $[\mathrm{N}$ ]. 深圳特区报，2014-04-08(A05).

Shenzhen Municipal Bureau of Statistics. Shenzhen national economic and social development statistical bulletin 2013 [N]. Shenzhen Special Economic Zone News, 2014-0408 (A05). (in Chinese)

[ 2 ] 深圳市统计局. 深圳市 2018 年国民经济和社会发展统 计公报 $[N]$ ．深圳特区报，2019-04-17(A05)。

Shenzhen Municipal Bureau of Statistics. Shenzhen national economic and social development statistical bulletin 2018 [ N ]. Shenzhen Special Economic Zone News, 2019-04-08 (A05). ( in Chinese)

[3] 范武波, 陈军辉, 钱 骏, 等. 机动车尾气对人体健康 的危害 $[\mathrm{J}]$. 中国环境管理, 2016, 8(1) : 110-113.

FAN Wubo, CHEN Junhui, QIAN Jun, et al. Effects of vehicle emissions on human's health $[\mathrm{J}]$. Chinese Journal of Environmental Management, 2016, 8 (1): 110-113. (in Chinese)

[ 4 ] HART J E, LADEN F, PUETT R C, et al. Exposure to traffic pollution and increased risk of rheumatoid arthritis [ J ]. Environmental Health Perspectives, 2009, 117 (7) : 1065-1069.

[ 5 ] 邱洪斌, 祝丽玲, 张风梅. 城市街道大气颗粒物污染特 征及影响因素的研究 $[\mathrm{J}]$. 黑龙江医药科学, 2002, 25 (3) : 3-4.

QIU Hongbin, ZHU Liling, ZHANG Fengmei. Pollution characteristics of atmospheric particles from city streets and influencing factors $[\mathrm{J}]$. Heilongjiang Medicine and Pharmacy, 2002, 25(3) : 3-4. (in Chinese)

[6] 谢 敏, 蔡 铭, 余 志, 等. 广州市主干道附近大气 污染物质量浓度的特征及影响因素研究 $[\mathrm{J}]$. 环境科 学与管理, 2008(3): 63-66, 149.

XIE Min, CAI Ming, YU Zhi, et al. Study on the characteristics and affecting factors of atmospheric pollution concentrations near an artery in Guangzhou $[\mathrm{J}]$. Environmental Science and Management, 2008 (3): 63-66, 149. (in Chinese)

[ 7 ] 陈天增, 葛艳丽, 刘永春, 等. 我国机动车排放 VOCs 及其大气环境影响 $[\mathrm{J}]$. 环境科学，2018，39(2)： 478-492.

CHEN Tianzeng, GE Yanli, LIU Yongchun, et al. VOCs emission from motor vehicles in China and its impact on the atmospheric environment $[\mathrm{J}]$. Environmental Science, 2018, 39(2) : 478-492. (in Chinese)

[8] 李慧颖. 应对机动车尾气排放污染的城市 “低排放区” 立法研究 $[D]$. 济南: 山东大学, 2014.
LI Huiyin. A legislative study on the urban low emission zone to deal with the motor vehicle exhaust pollution $[\mathrm{D}]$. Ji'nan: Shangdong University, 2014. (in Chinese)

[9]胡明伟，翟素云，段华波，等. 欧洲低排放区设置的 比较分析 $[J]$. 深圳大学学报理工版，2017，34(3)： 229-237.

HU Mingwei, ZHAI Suyun, DUAN Huabo, et al. Comparative analysis on the seeting of low emission zone in Europe $[\mathrm{J}]$. Journal of Shenzhen University Science and Engineering, 2017, 34(3) : 229-237. (in Chinese)

[10] HOLMAN C, HARRISON R M, QUEROL X. Review of the efficacy of low emission zones to improve urban air quality in European cities $[\mathrm{J}]$. Atmospheric Environment, 2015, 111: 161-169.

[11 ] BUCCOLIERI R, SANDBERG M, DI SABATINO S. City breathability and its Link to pollutant concentration distribution within urban-like geometries $[\mathrm{J}]$. Atmospheric Environment, 2010, 44(15) : 1894-1903.

[12 ] KIM J J, SMORODINSKY S, LIPSETT M, et al. Trafficrelated air pollution near busy roads the East Bay children's respairatory health study $[\mathrm{J}]$. American Jounal of Respairatory and Critical Care Medicine, 2004, 60(4) : 170-520.

[13] BERKOWICZ R, KETZEL M, VACHON $\mathrm{G}$, et al. Examination of traffic pollution distribution in a street canyon using the Nantes' 99 experimental data and comparison with model results $[\mathrm{J}]$. Water, Air, \& Soil Pollution, 2002, 2(5/6): 311-324.

[14 ] BERKOWICZ R, WINTHER M, KETZEL M. Traffic pollution modelling and emission data $[\mathrm{J}]$. Environmental Modelling \& Software, 2006, 21(4) : 454-460.

[15］袁 否, 宛 杨, 何 成. 基于 CFD 模拟的高密度街 区交通污染物分布 $[\mathrm{J}]$. 深圳大学学报理工版，2019， $36(3)$ : 274-280.

YUAN Lei, WAN Yang, HE Cheng. Distribution of traffic pollutants in high-density blocks based on CFD simulation $[\mathrm{J}]$. Journal of Shenzhen University Science and Engineering, 2019, 36(3) : 274-280. (in Chinese)

【中文责编：晨 兮; 英文责编：新 谷】 